\title{
Two-phase Flow Patterns in a Square Mini-channel
}

\author{
Jianfu ZHAO Gang LIU Bin LI \\ National Microgravity Laboratory, Institute of Mechanics, Chinese Academy of Sciences, Beijing 100080, \\ China.
}

\begin{abstract}
This paper presents a new set of experimental data of air-water flow patterns in a channel with a cross-section of $1 \times 1 \mathrm{~mm}^{2}$. The ranges of the gas and liquid superficial velocities are $0.1 \sim 10 \mathrm{~m} / \mathrm{s}$ and $0.2 \sim 7 \mathrm{~m} / \mathrm{s}$, respectively. Bubble, bubble-slug, slug, and frothy flows are observed. The present data are compared with other data in minichannels reported in literature, and also compared with those in normal channel at microgravity, in which the Bond number has the same order of magnitude. The slug-frothy boundary is in consistent with each other, but for the bubble-slug transition, a much smaller value for the transition quality in the drift-flux model is obtained in the present study than those predicted by the empirical relations for the case of microgravity. It's shown that the mini-scale modeling may not be an effective way to anticipate the bubble-slug transition of two-phase flow at microgravity.
\end{abstract}

Keywords: two-phase flow, flow pattern, mini-channel, microgravity

CLC number: V211.1 ${ }^{+} 7$ Document code: A Article ID: 1003-2169(2004)02-0174-05

\section{Introduction}

Two-phase gas-liquid flows are of great interest to the design of active thermal control system, power cycle, storage and transfer of cryogenic fluids, and other systems for spacecraft. Reliable design of such systems requires a thorough understanding of the mechanism of two-phase flow at microgravity, such as the phase distributions or flow patterns, the pressure drops and the heat transfer coefficients at different gas and liquid flow rates. These requirements of space applications have stimulated studies on two-phase flows at microgravity in the past decades ${ }^{[1]}$.

The ideal location to conduct these studies is aboard space station ${ }^{[2]}$ or in orbiting space shuttle ${ }^{[3]}$. However, due to high cost and limited accessibility, most of the experiments on two-phase flows at microgravity are performed using some ground-based facilities such as drop tower and parabolic aircraft. There exist some drawbacks to these ground-based experiments. For example, the residual accelerations less than $0.01 g_{0}$ are difficult to maintain on board parabolic aircraft. The gjitters of comparable order are often experienced. The overload of near $2 g_{0}$ and the rapid change in gravitational fields before and after each microgravity portion can induce undesired system transients. The very limited test duration (typically 20 seconds) makes it difficult to eliminate these transient effects. Moreover, for the sake of safety, only certain fiuids can be used. The cost is still high by comparison with tests on earth.

Mini-scale modeling on earth $^{[4]}$ is an inexpensive way to anticipate the behavior of two-phase flows at microgravity and to help defining needs for space or aircraft tests. The idea in the mini-scale modeling is to reduce the impact of gravity and increase that of surface tension. The ratio between these two forces is the Bond number $B o=\left(\rho_{\mathrm{L}}-\rho_{\mathrm{G}}\right) g d^{2} / \sigma$. Thus, reducing the diameter by a factor of 10 will result in reducing the Bond number by a factor of 100 , a change equivalent to reducing gravity by a factor of 100 as in the aircraft experiments.

On the other hand, capillary gas-liquid two-phase flows also occur in increasingly more modern industrial applications on earth. Studies generally confirm significant differences between capillaries and large channels, with respect to flow patterns ${ }^{[5 \sim 15]}$, pressure drop ${ }^{[9,16 \sim 20]}$, and boiling heat transfer and critical heat flux ${ }^{[16,21]}$. Criteria for determining the maximum channel diameter for channel orientation-independent (or, equivalently, gravity-independent) flow have also been proposed by Suo \& Griffith ${ }^{[5]}$, Brauner ${ }^{[22]}$, Brauner \& Moalem-Maron ${ }^{[23]}$, and Fukano \& Kariyasaki ${ }^{[9]}$. Zhao et al. ${ }^{[2]}$ found that the gravity-independent criterion 


\begin{tabular}{|c|c|c|c|}
\hline & Nomenclature & $U_{\mathrm{SL}}$ & the liquid superficial velocity \\
\hline Bo & the Bond number & $X c$ & the transition quality in drift-flux model \\
\hline$C_{0}$ & gas phase distribution parameter in drift-flux model & & Greek \\
\hline$d$ & diameter & $\varepsilon_{\text {cT }}$ & the critical void fraction in drift-flux model \\
\hline$g$ & gravity & $\mu$ & viscosity \\
\hline$g_{0}$ & gravity at sea level & $\rho$ & density \\
\hline $\operatorname{Re}$ & the Reynolds number & $\rho_{\mathrm{G}}$ & density of the gas phase \\
\hline$U$ & velocity & $\rho_{\mathrm{L}}$ & density of the liquid phase \\
\hline$U_{\mathrm{SG}}$ & the gas superficial velocity & $\sigma$ & surface tension \\
\hline
\end{tabular}

developed on the base of the experiments of two-phase gas-liquid flows at different gravity conditions aboard the Russian Mir Space Station is the same as that proposed by Brauner \& Moalem-Maron ${ }^{[23]}$, namely the Bond number is no more than a critical value of an order of 1 .

However, due to the extreme complication of twophase flows, some other dimensionless parameters, for example, the Reynolds number $R e=\rho U d / \mu$ which may be defined for the two-phase mixture or for each phase alone, should also play an important role. Therefore, prudence is needed in interpreting the experimental results of the mini-scale modeling. In the present paper, a new experiment of two-phase air-water flow patterns in a square mini-channel is performed. The experimental results are compared with those obtained at microgravity, in which the Bond number has the same order of magnitude. They are also compared with relevant flow pattern transition models, and some problems associated with the mini-scale modeling are discussed.

\section{Experimental Techniques}

A schematic diagram of the experimental facility is given in Fig.1.

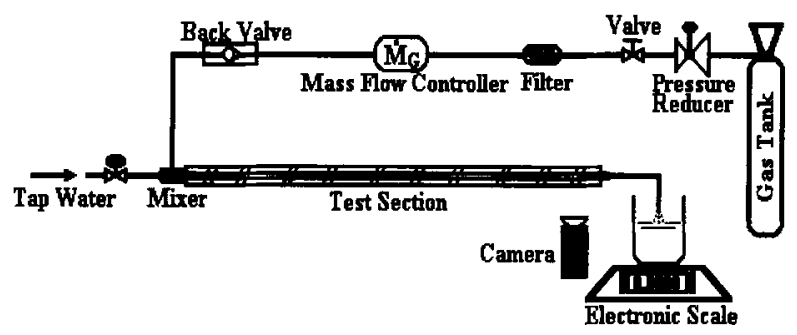

Fig. 1 Schematic diagram of the experimental facility

Fresh tap water and air were used as the experimental mediums. An electronic scale with a range of $0 \sim 1.0 \mathrm{~kg}$ and an accuracy of $10^{-5} \mathrm{~kg}$ was used to measure the flow quantity in certain duration (typically 200 seconds), which was measured using a stopwatch with an accuracy of 0.2 seconds. Air was stored in an 8-liter compressed air tank. After the pressure regulator the air pressure was reduced to $0.5 \mathrm{MPa}$. The outlet of the pressure regulator was connected to a mass flow controller. Two mass flow controllers, which have respectively range of $0 \sim 0.06$ SLM (standard liters per minute) and 0 0.6 SLM, were used to measure the flow rate of the gas phase. The accuracy is $1.5 \%$ FS (full scale) for both mass flow controllers.

A Pyrex glass prism was milled to form the square shaped channel along the length of the test section. A flat strip of Pyrex glass was used to create another side, or face, to the square shaped channel. The cross-sectional area of the channel is $1.00 \times 1.00 \mathrm{~mm}^{2}$. Its error was estimated to be within $\pm 8 \%$, which is major resource of the uncertainties of the measured superficial velocities of the gas and liquid phases.

A mixer is located before the test section. It is a cross component through which the test section passes. In the mixing chamber, gas flows into the test section through four holes, which are $0.7 \mathrm{~mm}$ in diameter, perpendicular to the channel. The distance between the mixer outlet and the outlet of the test section is $300 \mathrm{~mm}$. The test section is horizontal with respect to the gravity.

Prior to experiments, the test loop was carefully leak-tested with the highest liquid and air flow rates imposed to the system. After all leaks were eliminated, experiments were performed by imposing a nearly constant flow rate of tap water, while varying the flow rate of air. All experiments were performed at ambient temperature and near the atmospheric pressure.

The flow patterns in the test section were identified visually with the aid of a digital video camera recorder (SONY DCR-TRV900E). The camera was always targeted at the center of the test section. In order to eliminate the image blur, the highest shutter speed of the camera, namely 1/10000 second, was adopted in all experimental runs. The distance between the point pictured by the camera and the mixer outlet was larger than 200 channel "equivalent", or hydraulic, diameters everywhere. The entrance influence may be considered to be negligible, and thus the flow patterns observed can be considered to be fully developed.

\section{Flow Pattern Observations}

Four flow patterns, namely bubble, bubble-slug, slug, and frothy flows, are observed in the present experiments, 
in which the ranges of the gas and liquid superficial velocities $\left(U_{\mathrm{SG}}\right.$ and $\left.U_{\mathrm{SL}}\right)$ were $0.1 \sim 10 \mathrm{~m} / \mathrm{s}$ and $0.2 \sim 7$ $\mathrm{m} / \mathrm{s}$, respectively. The characteristics of these flow patterns are shown in Fig.2. Flows are all from right to left in the figure.

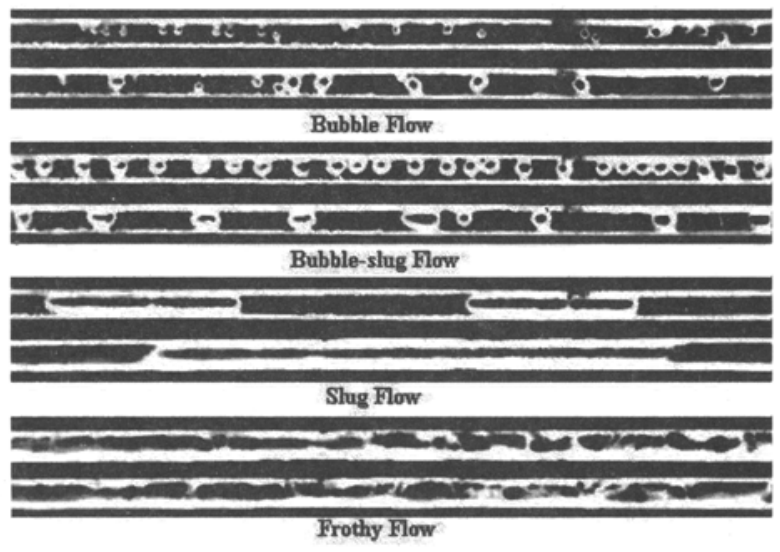

Fig. 2 Typical flow patterns observed in the mini-channel

Bubble flow characterized by distinct bubbles, occurs in relatively small gas superficial velocity. The shapes of the gas bubbles are not always spherical ones, but the equivalent diameters of the gas bubbles are always smaller than the side length of the test channel. With increasing $U_{\mathrm{SG}}$ (which leads to increasing the void fraction), the gas bubbles became crowded and their size increased, which eventually led to the development of the slug flow.

Slug flow is characterized by elongated cylindrical bubbles with smooth interfaces. For those near the bubble-slug transition boundary, the length of most gas bubble is only slightly bigger than two times of the side length of the test channel. The nose of these bubbles always has slightly pronounced conical shape along the flow direction, while the rear remains the spherical shape. Small gas bubble can still be observed in these flows. Parameter changes leading to higher void fraction (e.g. increasing $U_{\mathrm{SG}}$ or/and decreasing $U_{\mathrm{SL}}$ ), lead to longer bubbles, while small, axisymmetric disturbances may exist in the interface. At much higher $U_{\mathrm{SG}}$, the rear of the elongated bubbles will lose its $\mathrm{s}$ spherical shape firstly. Large, non-axisymmetric disturbances will appear in the interface at even higher $U_{\mathrm{SG}}$.

Frothy flow occurs at much higher $U_{\mathrm{SG}}$. The interface between the gas and liquid phases becomes more irregular and disruption always occurs. Some frothy structures, which result in some dim shadows, appear occasionally in the test channel. It is observed firstly in the present experiments that the liquid phase can form irregular drops moving along the top or bottom side (maybe the corner) of the test channel. There is also bubble-slug flow identified in several experimental runs.
In these flows, most of the gas bubbles are smaller than the side length of the channel while elongated gas bubbles with the length not more than two times of the side length of the test channel can also be observed infrequently. The interval between two elongated bubbles is relatively large.

According to the above observations, a flow pattern map is presented in Fig.3.

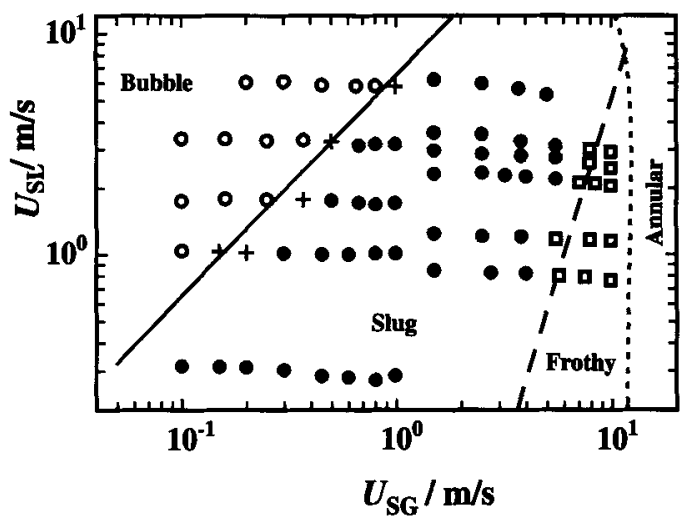

Fig.3 Flow pattern map

bubble, + bubble-slug, $\bigcirc$ slug, $\square$ frothy

- Drift-flux model with $X_{c}=0.133$

- - Empirical Weber model for slug-frothy transition ${ }^{\text {[30] }}$

... semi-theoretical Weber model for slug-annular transition ${ }^{[28]}$

\section{Discussion}

Firstly, we compare the observed bubble-slug transition boundary in the present experiment with those of Triplett et al. ${ }^{[12]}$ obtained with their $1.1 \mathrm{~mm}$-hydraulic diameter circular and semi-triangular test sections (Fig.4). It shows a relatively good agreement, which indicates that there may exist no influence of the cross-sectional shape on this transition.

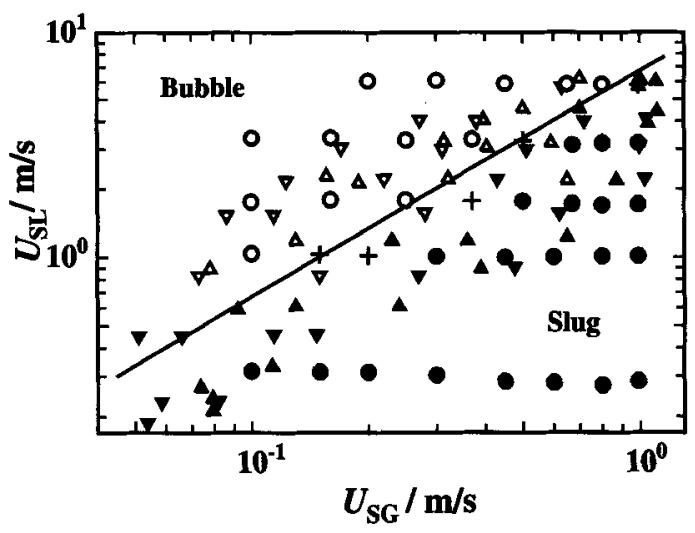

Fig.4 The bubble-slug transition

Square channel (present): $O$ bubble, + bubble-slug, 1 slug

Circular channel ${ }^{[12]}: \triangle$ bubble, $\Delta$ slug

Semi-triangular channel ${ }^{[12]}: \nabla$ bubble, $\nabla$ slug

- Drift-flux model with $X_{c}=0.133$ 
Based on the comparisons made by Triplett et al. ${ }^{[12]}$, i.e. Figs.10 and 11 in their paper, the present data are also consistent with those of Fukano \& Kariyasaki $^{[9]}$ obtained with their $1 \mathrm{~mm}$-diameter circular test section, while large differences will be found between the present data and those of Damianides \& Westwater ${ }^{[8]}$. The inconsistencies associated with the identified flow patterns, which can be inferred from the denominations of flow patterns used by different authors, may be part of the reasons resulting in these differences.

The most frequently used model for predicting the bubble-slug transition in normal size channel at microgravity is the drift-flux model ${ }^{[25,26]}$, which can be expressed as:

$$
U_{S L}=U_{S G}\left(1-X_{c}\right) / X_{c}
$$

where the transitional quality $X_{c}=C_{0} \varepsilon_{c r}$. Two empirical correlations for the transitional quality were proposed separately by Colin et al. ${ }^{[26]}$ and Jayawardena et al. ${ }^{[27]}$, based on the same database of two-phase flows in normal size channel at microgravity. Detailed comment on them can be found in Zhao's paper ${ }^{[1]}$.

Assuming the drift-flux model is still active in capillary two-phase flows, a value of 0.133 for the transitional quality can be obtained from the present experimental data (Fig.3 or 4). This value is much smaller than those obtained at microgravity. For example, a value of 0.44 for the transitional quality was obtained by Zhao et al. ${ }^{[28]}$, which also satisfy the correlation of Jayawardena et al. ${ }^{[27]}$. The experiments of Zhao et al. ${ }^{[28]}$ were performed in a square channel with a cross-section of $12 \times 12 \mathrm{~mm}^{2}$ aboard the Russian IL-76 reduced gravity airplane. The value of the Bond number is about 0.58 in the flight experiment, while $B o=0.136$ in the present experiment. They are of the same order, under which two-phase flows are gravity-independent. According to the correlation of Jayawardena et al. ${ }^{[27]}$, a value of 0.8 should be taken for the transitional quality in the present study, which is much more than 0.133 , the value based on the experimental data.

The difference between the experimental values of the transitional quality in the two cases may indicate that there should be different mechanisms underlying the bubbleslug transition in the capillary two-phase flow and twophase flow in normal size channel at microgravity. Thus, mini-scale modeling could not be an effective way to anticipate the behavior of (at least) the bubble-slug transition in normal size channel at microgravity.

The present experimental data for higher gas superficial velocity are also in satisfactory overall agreement with similar experimental data report in the literature, when inconsistencies associated with the identified flow patterns are removed. However, it can't be concluded that there isn't influence of the cross- sectional shape on the flow patterns and their transitions due to the subjectivity of the flow pattern identification used in the present study and others. Methods that can accurately and objectively determine the flow patterns are highly desirable, especially for the cases of high flow rate.

In Fig.3, the present experimental data are compared with the prediction of the semi-theoretical Weber number model, which was proposed for circular channels by $\mathrm{Zhao} \& \mathrm{Hu}^{[29]}$ and modified by Zhao et al. ${ }^{[28]}$ to conclude the shape influence of the test section. It has been verified that the predictions of this model are in good agreement with the data obtained from the twophase flow experiments at microgravity and the miniscale or/and neutral-buoyancy modeling at normal gravity environment. No contradiction between the prediction and the present experimental data is observed. However, since no annular flow was observed in the limited ranges of the flow rates of two phases, the actual transition to the annular flow, which will occur in the same system for larger gas superficial velocities, was not obtained in the present study.

The boundary between slug and frothy flows at microgravity determined experimentally by Lowe \& Rezkallah $^{[30]}$ is also plotted in Fig.3. The value of the Bond number in their study is about 0.36 . It shows that this transition line is close to the slug-frothy transition boundary observed in the present study. It may indicate that the mini-scale modeling can be used to anticipate the behavior of the two-phase flow with high flow rates in normal size channel at microgravity. Further studies, however, are still needed in order to make correct interpretation of the results of the modeling.

\section{Conclusions}

A new set of experimental data of two-phase air-water flow patterns in a square mini-channel is presented. The channel has a cross-section of $1 \times 1 \mathrm{~mm}^{2}$ and a length of $300 \mathrm{~mm}$. The ranges of the gas and liquid superficial velocities are $0.1 \sim 10 \mathrm{~m} / \mathrm{s}$ and $0.2 \sim 7 \mathrm{~m} / \mathrm{s}$, respectively. Four kinds of flow patterns, namely bubble, bubble-slug, slug, and frothy flows, are observed. The experimental results are compared with the similar experimental data reported in the literature, as well as those obtained from microgravity experiments, in which the Bond number is of the same order as that in the present study. It has shown that the mini-scale modeling can be used to anticipate the behavior of the two-phase flows with high flow rates in normal size channel at microgravity, while it may be not an effective way for the bubble-slug transition.

\section{Acknowledgements}

The authors would like to acknowledge the financial 
support provided by the National Natural Science Foundation of China (10202025) and the Knowledge Innovation Program of Chinese Academy of Sciences (KJCX2-SW-L05).

\section{References}

[1] Zhao, J F. A Review of Two-phase Gas-liquid Flow Patterns under Microgravity Conditions. Adv. Mech., 1999, 29(3): 369-382

[2] Zhao, J F, Xie, J C, Lin, H, et al. Experimental Studies on Two-Phase Flow Patterns Aboard the Mir Space Station. Int. J. Multiphase Flow, 2001, 27(11): 1931-1944

[3] Reinarts, T R, Ungar, E K, Butler, C D. Adiabatic TwoPhase Pressure Drop in Microgravity: TEMP2A-3 Flight Experiment Measurements and Comparison with Predictions. 33 ${ }^{\text {rd }}$ Aerospace Sciences Meeting \& Exhibit, Reno, NV, USA, 1995

[4] Galbiati, L, Andreini, P. Flow Pattern Transition for Horizontal Air-Water Flow in Capillary Tubes, A Microgravity "Equivalent System" Simulation. Int. Commun. Heat Mass Transfer, 1994, 21(4): 461 -468

[5] Suo, M, Griffith, P. Two-phase Flow in Capillary Tubes. Trans ASME J. Basic Eng., 1964, 86(3): 576-582

[6] Oya, T. Upward Liquid Flow in Small Tube Into Air Streams. Bulletin of JSME, 1971, 14(78): 1320-1339

[7] Barnea, D, Luninski, Y, Taitel, Y. Flow Pattern in Horizontal and Vertical Two Phase Flow in Small Diameter Pipes. Can. J. Chem. Eng., 1983, 61(5): 617620

[8] Damianides, C A, Westwater, J W. Two-phase Flow Patterns in a Compact Heat Exchanger and in Small Tubes. $2^{\text {nd }}$ UK Nat. Conf. on Heat Transfer, Glasgow, UK, 1988

[9] Fukano, T, Kariyasaki, A. Characteristics of Gas-liquid Two-phase Flow in a Capillary Tube. Nucl. Eng. Des., 1993, 141(1-2): 59-68

[10] Barajas, A M, Panton, R L. The Effects of Contact Angle on Two-Phase Flow in Capillary Tubes. Int. J. Multiphase Flow, 1993, 19(2): 337-346

[11] Mishima, K, Hibiki, T. Some Characteristics of AirWater Two-Phase Flow in Small Diameter Vertical Tubes. Int. J. Multiphase Flow, 1996, 22(4): 703-712

[12] Triplett, K A, Ghiaasiaan, S M, Abdel-Khalik, S I, et al. Gas-liquid Two-phase Flow in Microchannels. Part I: Two-phase Flow Patterns. Int. J. Multiphase Flow, 1999, 25(3): 377-394

[13] Coleman, J W, Garimella, S. Characterization of Twophase Flow Patterns in Small Diameter Round and Rectangular Tubes. Int. J. Heat Mass Transfer, 1999, 42(15): $2869-2881$

[14] Zhao, T S, Bi, Q C. Co-current Air-water Two-phase Flow Patterns in Vertical Triangular Microchannels. Int. J. Multiphase Flow, 2001, 27(5): 765-782

[15] Yang, C-Y, Shieh, C-C. Flow Pattern of Air-Water and
Two-Phase R-134a in Small Circular Tubes. Int. J. Multiphase Flow, 2001, 27(7): 1163-1177

[16] Inasaka, F, Nariai, H, Shimura, T. Pressure Drops in Subcooled Flow Boiling in Narrow Tubes. Heat Transfer Jap. Res., 1989, 19(1): 70-82

[17] Lin, S, Kwok, C C K, Li, R-Y, et al. Local Frictional Pressure Drop During Vaporization of R-12 through Capillary Tubes. Int. J. Multiphase Flow, 1991, 17(1): 95- 102

[18] Fouran, M, Bories, S. Experimental Study of Air-Water Two-Phase Flow Through a Fracture (Narrow Channel). Int. J. Multiphase Flow, 1995, 21(4): 621-637

[19] Triplett, K A, Ghiaasiaan, S M, Abdel-Khalik, S I, et al. Gas-Liquid Two-Phase Flow in Microchannels. Part II: Void Fraction and Pressure Drop. Int. J. Multiphase Flow, 1999, 25(3): 395-410

[20] Zhao, T S, Bi, Q C. Pressure Drop Characteristics of GasLiquid Two-Phase Flow in Vertical Miniature Triangular Channels. Int. J. Heat Mass Transfer, 2001, 44(13): 2523-2534

[21] Peng, X F, Wang, B X. Forced Convection and Flow Boiling Heat Transfer for Liquid Flowing Through Microchannels. Int. J. Heat Mass Transfer, 1993, 36(14): $3421-3427$

[22] Brauner, N. On the Relations Between Two-Phase Flows under Reduced Gravity and Earth Experiment. Int. Commun. Heat Mass Transfer, 1990, 17(3): 271-282

[23] Brauner, N, Moalem-Maron, D. Identification of the Range of Small Diameter Conduits, Regarding Two-Phase Flow Pattern Transitions. Int. Commun. Heat Mass Transfer, 1992, 19(1): 29-39

[24] Zhao, J F, Xie, J C, Lin, H, et al. Experimental Study of Two-Phase Flow in Microgravity. $51^{\text {st }}$ Int. Astronautical Cong., Rio De Janeiro, Brazil, 2000

[25] Dukler, A E, Fabre, J A, Mcquillen, J B, et al. Gas-Liquid Flow at Microgravity Conditions: Flow Patterns and Their Transitions. Int. J. Multiphase Flow, 1988, 14(4): 389400

[26] Colin, C, Fabre, J, McQuillen J. Bubble and Slug Flow at Microgravity Conditions: State of Knowledge and Open Questions. Chem. Eng. Comm., 1996, 141/142: 155-173

[27] Jayawardena, S S, Balakotaiah, V, Witte, L C. Flow Pattern Transition Maps for Microgravity Two-Phase Flows. Aiche J., 1997, 43(6): 1637-1640

[28] Zhao, J F, Xie, J C, Lin, H, et al. Experimental Study on Two-phase Gas-liquid Flow Patterns at Normal and Reduced Gravity Conditions. Science in China, Ser. E, 2001, 44(5): 553-560

[29] Zhao, J F, Hu, W R. Slug to Annular Flow Transition of Microgravity Two-Phase Flow. Int. J. Multiphase Flow, 2000, 26(8): 1295-1304

[30] Lowe, D C, Rezkallah, K S. Flow Regime Identification in Microgravity Two-phase Flows Using Void Fraction Signals. Int. J. Multiphase Flow, 1999, 25(3): 433-457 